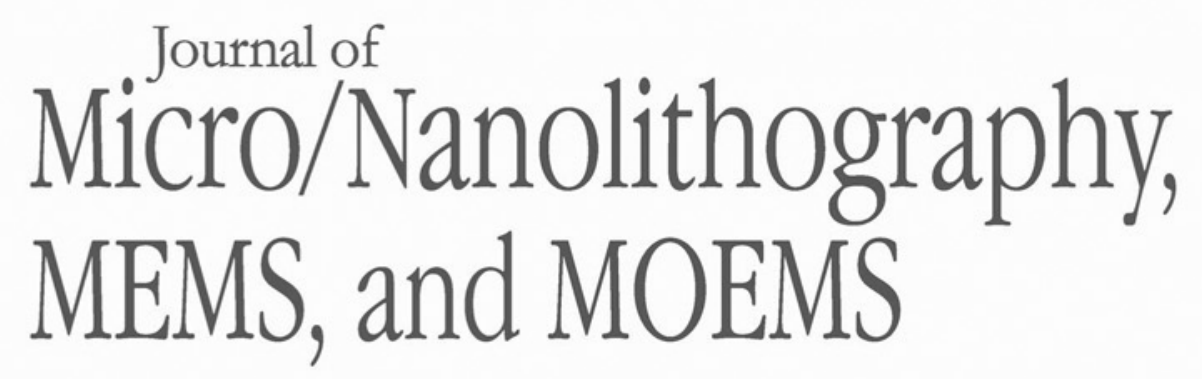

Nanolithography.SPIEDigitalLibrary.org

\title{
JM3 2014 List of Reviewers
}

\section{SPIE.}


The Journal of Micro/Nanolithography, MEMS, and MOEMS would like to sincerely thank the following individuals who served as reviewers in 2014 . The success of our publication hinges on the voluntary contributions of time and energy put forth by these professionals.

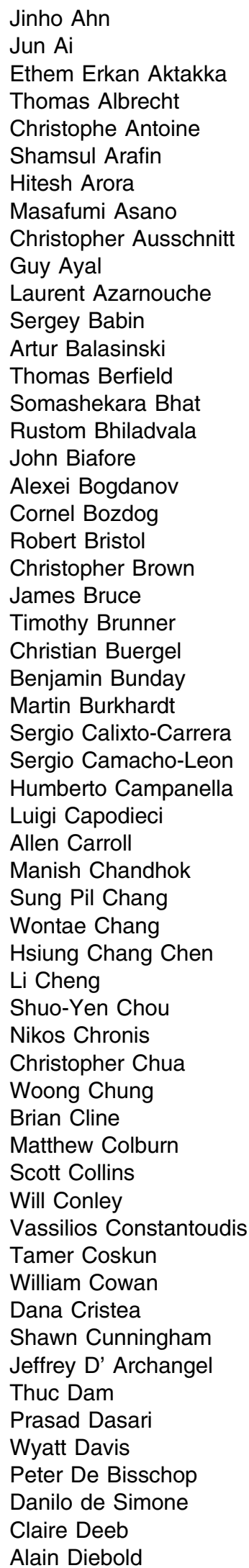

Jinho Ahn

Thomas Albrecht

Christophe Antoine

Shamsul Arafin

Christopher Ausschnitt

Guy Ayal

Laurent Azarnouche

Thomas Berfield

Somashekara Bhat

John Biafore

Alexei Bogdanov

Christopher Brown

James Bruce

Timothy Brunner

Christian Buergel

Benjamin Bunday

Sergio Calixto-Carrera

Sergio Camacho-Leon

Humberto Campanella

Luigi Capodieci

Allen Carroll

anish Chandhok

Wontae Chang

Hsiung Chang Chen

Li Cheng

Shuo-Yen Chou

Nikos Chronis

Christopher Chua

Brian Cline

Matthew Colburn

Scott Collins

Will Conley

Vassilios Constantoudis

Tamer Coskun

William Cowan

Dana Cristea

Thuc Dam

Prasad Dasari

Alain Diebold
Lars Dittrich

Elizabeth Dobisz

Huihang Dong

Ted Dziura

Andrew Eckert

Bodo Ehlers

Christopher Ellison

Ilan Englard

Andreas Erdmann

Li Fan

Yongfa Fan

Jeff Farnsworth

Nelson Felix

Jo Finders

Igor Fomekov

Carlos Fonseca

Johann Foucher

Lynn Fuller

Emily Gallagher

Gregg Gallatin

Bernd Geh

Yourdan Georgiev

Thomas Gessner

Rani Ghaida

Reza Ghodssi

Valeriy Ginzburg

Lynford L. Goddard

Poonam Goel

Evangelos Gogolides

Frank Goodwin

Yuri Granik

Trey Graves

Roel Gronheid

L. Jay Guo

Puneet Gupta

Scott Halle

Tetsuo Harada

Carsten Hartig

Naoya Hayashi

Yuan $\mathrm{He}$

Scott Hector

Eric Hendrickx

Pedro Herrera

Michael S. Hibbs

Tatsuhiko Higashiki

Mark Horn

Stephen Hsu

Der-Ray Huang

Xian Huang

Phillip Hustad

Kim Jaehwan

Chris Jones

Ronald Jones

Prasoon Joshi

Aju Jugessur

II Woong Jung

Young-Seog Kang
Tolga Kaya

Adrian Keating

Ajit Khosla

Jungkwun kim

Seok-min Kim

SeongSue Kim

Seunghyun Kim

Young-Chang Kim

Edward Kinzel

Katherine Kirk

Ulrich Klostermann

Sachiko Kobayashi

Juergen Koch

Shinichi Kojima

Hareesh Komepalli

Konstantin Koshelev

Ramakrishna Kotlanka

Minoru Kurosawa

Steffen Kurth

Jongwook Kye

Neal Lafferty

Kafai Lai

Edmund Lam

Azat Latypov

Richard Lawson

Bertrand Le-Gratiet

Ming-Chang Lee

Ralf Lenigk

Shimon Levi

Hongqiang $\mathrm{Li}$

Kary Li

Pei Li

Peng Li

Ted Liang

Lars Liebmann

Dmitriy Likhachev

Chenxi Lin

Yu-Sheng Lin

Anna Lio

Chi Chun Liu

Guoliang Liu

Hewei Liu

Robin Liu

Weijun Liu

Ye Liu

Zewen Liu

Chiung-Cheng Lo

Xu Ma

Chris Mack

Maurine Malak

Matt Malloy

John Maltabes

Pawitter Mangat

Andrew Mason

Kiron Mateti

Lawrence Melvin, III

Zhengyu Miao 
Marshal Miller

Michael Miller

Hiroyuki Miyazoe

Hakaru Mizoguchi

Mohammad Moghimi

Waleed Mohammed

Lars Montelius

Swamy Muddu

Jit Muthuswamy

Seiji Nagahara

Mark Neisser

Jens Timo Neumann

Andrew Neureuther

Patricia Nieva

Honseok Noh

Tatiana Novikova

Rekha Pai

Nezih Pala

Liang Pan

Ashok Pandey

Hardik Pandya

Linyong Pang

Erwine Pargon

Dongwoon Park

Sebastien Pauliac-Vaujour

Marcus Pelegrini

Jan Hendrik Peters

Brennan Peterson

Carlos Pina

Jed Pitera

Michael Postek

Moshe Preil

Ananthan Raghunathan

Mina Rais-Zadeh

Abelardo Ramirez-Hernandez

Narender Rana

Jed Rankin

Atabak Rashidian

Abbas Rastegar

Christopher Raymond
Kailiang Ren

Balusamy Renganathan

Michael Rieger

Paulina Rincon Delgadillo

Tony Rogers

Julia Sakamoto

Andrew Sarangan

Issei Sasaki

Tarun Saxena

Hella-Christin Scheer

Bernd Schulz

Matthew Sendelbach

Debbie Senesky

Alexander Serebryakov

Charles Settens

Guocheng Shao

Mohammad Shavezipur

Li Shen

Masato Shibuya

Tsutomu Shoki

Sameet Shriyan

Richard Silver

Mark D. Smith

Nigel Smith

Robert Socha

Eric Solecky

Ian Stobert

Carlos Strocchia-Rivera

Chris Strolenberg

Yugang Sun

Mohamed Tahmaspur

Niall Tait

Kiwamu Takehisa

Yuksel Temiz

Tsuneo Terasawa

Fred Terry Jr.

Bradley Thiel

Micahel Thier

Albert Titus

Massimo Tormen
Juan Andres Torres

Van Truskett

Julius Tsai

Kevin Turner

Vladimir Ukraintsev

Alessandro Vaglio Pret

Kaushik Vaidyanathan

Anton van Oosten

Jan van Schoot

Leo Varghese

John Villarrubia

Andras Vladar

Curt Vogel

Marko Vogler

Pradeep Vukkadala

Kevin Walsh

Bin Wang

Jing Wang

Lidai Wang

Youmin Wang

Jim Wiley

ChinCheng Wu

Joyce Wu

Wei Wu

Xiaofei Wu

Yuan Xie

Ning Xue

Fuqian Yang

Kenji Yoshimoto

Todd Younkin

Kyoungsik Yu

Chi-min Yuan

Lei Zhai

Siyang Zheng

Chuanhong Zhou 